\title{
Some Gastrointestinal Function Regulatory Kampo Medicines Have Modulatory Effects on Human Plasma Adrenocorticotropic Hormone and Cortisol Levels with Continual Stress Exposure
}

\author{
Takafumi NaIto, * Hiroki Iтон, and Masaharu TakeYama \\ Department of Clinical Pharmacy, Oita Medical University; Hasama-machi, Oita 879-5593, Japan. \\ Received June 20, 2002; accepted October 10, 2002
}

\begin{abstract}
Rikkunshi-to, a gastrointestinal function regulatory traditional Chinese herbal (Kampo) medicine, has recently been evaluated for its clinical usefulness in stress and depression. This medicine has modulatory effects on the hypothalamo-pituitary-adrenal axis and autonomic nervous function. We examined the effect of Rikkunshi-to and the other gastrointestinal function regulatory Kampo medicines, Hange-shashin-to, Hange-koboku-to, and Ninjin-to, on the plasma levels of adrenocorticotropic hormone (ACTH) and cortisol under stress conditions by repetitive blood sampling. Rikkunshi-to, Hange-shashin-to, and Hange-koboku-to significantly suppressed increases in plasma ACTH-immunoreactive substance (IS) levels compared with the response to a placebo. Rikkunshi-to and Hange-shashin-to significantly suppressed increases in plasma cortisol levels compared with the response to placebo. Ninjin-to had no significant effect on plasma ACTH-IS and cortisol levels. In this study, Rikkunshi-to, Hange-shashin-to, and Hange-koboku-to (partially) regulated plasma ACTH and cortisol levels under stress. These modulatory effects might be beneficial in stress-related disease and suggest that these medicines have clinical pharmacologic activity.
\end{abstract}

Key words adrenocorticotropic hormone (ACTH); cortisol; Kampo; stress

Traditional Chinese herbal (Kampo) medicines (Rikkunshi-to, Hange-shashin-to, Hange-koboku-to, Ninjin-to, etc.) have frequently been used in the empirical treatment of chronic hypofunction of the gastrointestinal system. Some patients who take these Kampo medicines do not have organic diseases such as peptic ulcer, reflex esophagitis, and stomach cancer but have conditions classified as non-ulcer dyspepsia (NUD). ${ }^{1)}$ Most NUD patients tend toward depressive and psychosomatic conditions and are exposed to continual affective stress. ${ }^{2)}$ That continual stress causes abnormalities in the hypothalamo-pituitary-adrenal (HPA) axis and autonomic nervous function. ${ }^{3,4)}$

Rikkunshi-to, a gastrointestinal function regulatory Kampo medicine, has recently been evaluated for its clinical usefulness in stress and depression. ${ }^{5-7)}$ This medicine has modulatory effects on the HPA axis and autonomic nervous function and regulates adrenocorticotropic hormone (ACTH) and cortisol levels in plasma to normal ranges in NUD. ${ }^{8,9}$ Some abnormalities of gastrointestinal function are presumed to result from changes in hormone levels.

$\mathrm{ACTH}$ is a peptide containing 39 amino acids, and ACTHimmunoreactive substances (ACTH-IS) are found in tissue other than the pituitary gland (i.e., brain, adrenal gland, gastrointestinal tract, pancreas, thyroid gland, and placenta). ${ }^{10)}$ The secretion of ACTH is controlled by the mechanism of circadian rhythm and negative feedback from plasma cortisol and neurogenic stimulation. The peptide has the secretomotory action of glucocorticoid and becomes elevated under stress. Cortisol, commonly used to indicate the level of stress, is secreted by the zona fasciculata of the adrenal cortex and its secretion is dependent on the ACTH level.

In general, venipuncture for blood sampling is postulated to be a stress factor that can increase circulating ACTH and cortisol levels, etc. ${ }^{11,12)}$ Repetitive blood sampling places subjects under artificial stress, and venipuncture as a stressor is useful for the evaluation of the pharmacological effects of drugs. ${ }^{13-15)}$ Thus we examined the effect of four Kampo medicines on hormone levels (ACTH and cortisol) under continual stress.

\section{MATERIALS AND METHODS}

Materials Rikkunshi-to (EK-43, lot 26L99), prepared as a 4.1-g dried powder extract of Ginseng Radix (4.0 g), Atractylodis Rhizoma (4.0 g), Hoelen ( $4.0 \mathrm{~g})$, Pinelliae Tuber ( $4.0 \mathrm{~g})$, Aurantii Nobilis Pericarpium (2.0 g), Zizyphi Fructus ( $2.0 \mathrm{~g})$, Glycyrrhizae Radix (1.0 g), and Zingiberis Rhizoma $(0.5 \mathrm{~g})$; Hange-shashin-to (EK-14, lot 011A), prepared as a 3.8 -g dried powder extract of Pinelliae Tuber (5.0 g), Scutellariae Radix (2.5 g), Zingiberis Rhizoma $(2.5 \mathrm{~g})$, Ginseng Radix (2.5 g), Glycyrrhizae Radix (2.5 g), Zizyphi Fructus $(2.5 \mathrm{~g})$, and Coptidis Rhizoma (1.0 g); Hange-koboku-to (EK-16, lot 27A09), prepared as a 1.5-g dried powder extract of Pinelliae Tuber $(6.0 \mathrm{~g})$, Hoelen $(5.0 \mathrm{~g})$, Magnoliae Cortex $(3.0 \mathrm{~g})$, Perillae Herba $(2.0 \mathrm{~g})$, and Zingiberis Rhizoma $(1.3 \mathrm{~g})$; and Ninjin-to (EK-32, lot 01BJ), prepared as a 3.0-g dried powder of Ginseng Radix (3.0 g), Glycyrrhizae Radix $(3.0 \mathrm{~g})$, Atractylodis Rhizoma (3.0 g), and Zingiberis Siccatum Rhizoma (3.0 g), were kindly supplied by Kanebo Co., Ltd. (Tokyo, Japan). The placebo was the additive of the above formulations alone.

Synthetic human ACTH (1-24) was purchased from the Peptide Institute (Osaka, Japan). Antiserum to human ACTH (A516/R1H) was purchased from Biogenesis (Newfields, U.K.). The TDx Cortisol assay kit was purchased from Dainabot (Tokyo, Japan). All other reagents were of reagent grade and commercially available.

Subjects Five healthy male volunteers (nonsmokers), aged 23-40 years, participated in this study. Each subject received information on the scientific purpose of the study, which was approved by the Ethics Committee of Oita Medical University, and gave written informed consent. No sub- 
ject received any medication for at least 1 month before the study, and there were at least 3-month intervals between other studies. The volunteers had participated in more than three similar studies.

Study Schedule Rikkunshi-to, Hange-shashin-to, Hangekoboku-to, or Ninjin-to at a dose of $6.0 \mathrm{~g}$ was administered orally. The same dose of placebo was also given to the volunteers. Venous blood samples from a forearm vein were taken before and $20-240 \mathrm{~min}$ after administration of the drug (eight times). All subjects ate lunch at 11:45-12:00, and the study was carried out from 14:00 until 18:00. In the study in which blood samples were taken at intervals of $120 \mathrm{~min}$, sampling was performed at 14:00, 16:00, and 18:00 without administering a test medicine.

Enzyme Immunoassay of ACTH The blood samples were placed in chilled tubes containing 500 kallikrein inhibitor units $/ \mathrm{ml}$ of aprotinin and $1.2 \mathrm{mg} / \mathrm{ml}$ of EDTA. After centrifugation, plasma samples were diluted with $4 \%$ acetic acid, $\mathrm{pH}$ 4.0, and loaded onto Sep-Pak C18 cartridges (Millipore, Milford, MA, U.S.A.). The peptides in plasma were eluted with $70 \%$ acetonitrile in $0.5 \%$ acetic acid, $\mathrm{pH} 4.0$, lyophilized, and stored at $-40{ }^{\circ} \mathrm{C}$ until assayed.

Peptide levels in plasma were measured using a sensitive enzyme immunoassay for ACTH-IS as previously described. ${ }^{16)}$ Human ACTH $(1-24)$ was conjugated with $\beta$-Dgalactosidase (Boehringer Mannheim, Mannheim, Germany) with $N$-( $\varepsilon$-maleimidocaproyloxy $)$-succinimide according to the method of Kitagawa et al. ${ }^{17}$ ) The assay was performed using a delayed addition method. Separation of bound and free antigen was performed on an anti-rabbit IgG (55641) (ICN Pharmaceuticals, Aurora, OH, U.S.A.)-coated immunoplate (Nunc-Immuno Module Maxisorp F8, InterMed, Denmark). The concentration of ACTH was expressed as ACTH (1-24), which has the biological activity of ACTH. The EIA for ACTH was specific and highly sensitive to detection limits of $2 \mathrm{fmol} / \mathrm{ml}$.

Determination of Plasma Cortisol Levels Plasma cortisol levels were measured using a fluorescence polarization immunoassay. The detection limit of cortisol was $6.4 \mathrm{ng} / \mathrm{ml}$. This method shows minimal cross-reactivity with the endogenous steroids (11-deoxycortisol [9.9\%], corticosterone $[6.3 \%]$, and others $[<3 \%])^{18)}$

Data Analysis ACTH-IS levels in plasma are expressed as mean \pm S.D. (pg/ml). Cortisol levels in plasma are expressed as concentration of mean \pm S.D. ( $\mathrm{ng} / \mathrm{ml}$ ). Comparisons of mean values were made by analysis of variance and Dunnett's test. A value of $p<0.05$ was regarded as significant.

\section{RESULTS}

Effects of Rikkunshi-to, Hange-shashin-to, Hangekoboku-to, and Ninjin-to on Plasma ACTH-IS Levels The plasma ACTH-IS level-time profile when blood was sampled at intervals of $120 \mathrm{~min}$ is shown in Fig. 1 (dotted line). The levels of ACTH-IS in samples at 120-min intervals $(4.3 \pm 2.0 \mathrm{pg} / \mathrm{ml}$ at $120 \mathrm{~min}, 5.3 \pm 2.2 \mathrm{pg} / \mathrm{ml}$ at $240 \mathrm{~min})$ showed suppression of increases compared with placebo, which reflected the effects of repetitive blood sampling.

Figure 1A shows the profiles of plasma ACTH-IS levels against time after the administration of Rikkunshi-to.
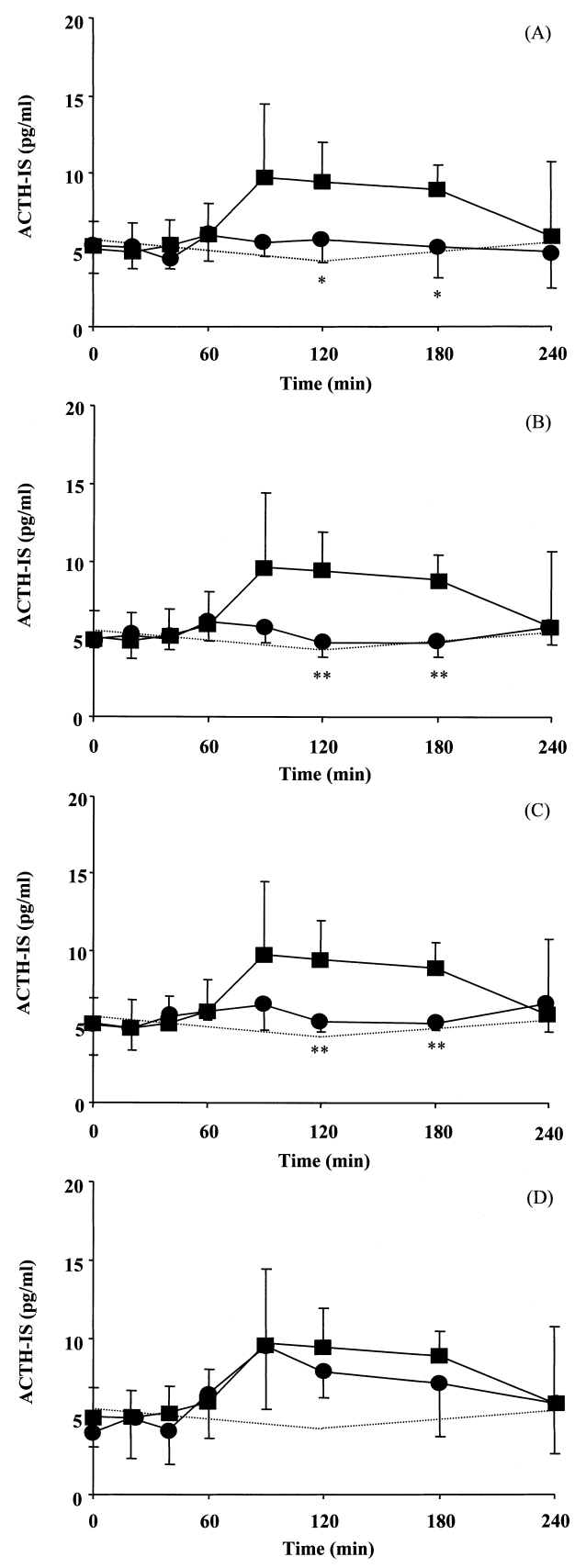

Fig. 1. Effect of Rikkunshi-to (A), Hange-shashin-to (B), Hange-kobokuto (C), and Ninjin-to (D) (O) or Placebo (⿶) on Plasma ACTH Levels

The dotted line represents the ACTH-IS levels when blood samples were taken at intervals of $120 \mathrm{~min}$. Each point represents the mean \pm S.D. of concentrations in five volunteers. $* * p<0.01, * p<0.05$ compared with the placebo group.

Rikkunshi-to significantly suppressed increases in ACTH-IS at 120 and $180 \mathrm{~min}$ compared with the response of the placebo. The ACTH-IS levels in volunteers after the administration of Rikkunshi-to were almost the same as those in samples taken at intervals of $120 \mathrm{~min}$.

Hange-shashin-to significantly suppressed increases in ACTH-IS at 120 and 180 min similar to Rikkunshi-to (Fig. 1B), and the effects of Hange-shashin-to were also similar to those of Hange-koboku-to (Fig. 1C).

Ninjin-to had no significant effect on plasma ACTH-IS (Fig. 1D). The levels of plasma ACTH-IS with Ninjin-to administration showed a tendency to increase compared with levels when blood was sampled at intervals of $120 \mathrm{~min}$. 
Effects of Rikkunshi-to, Hange-shashin-to, Hangekoboku-to, and Ninjin-to on Plasma Cortisol Levels Figure 2 (dotted line) shows the plasma cortisol level-time profile when blood samples were taken at intervals of $120 \mathrm{~min}$. Placebo caused significant increases in cortisol at $240 \mathrm{~min}$ compared with the levels when samples were taken at intervals of $120 \mathrm{~min}(58.8 \pm 10.4 \mathrm{ng} / \mathrm{ml})$, and no significant changes were seen at $120 \mathrm{~min}(90.0 \pm 30.1 \mathrm{ng} / \mathrm{ml})$.

Rikkunshi-to significantly suppressed increases in cortisol at 180 and 240 min compared with the response with placebo (Fig. 2A). Hange-shashin-to significantly suppressed in-
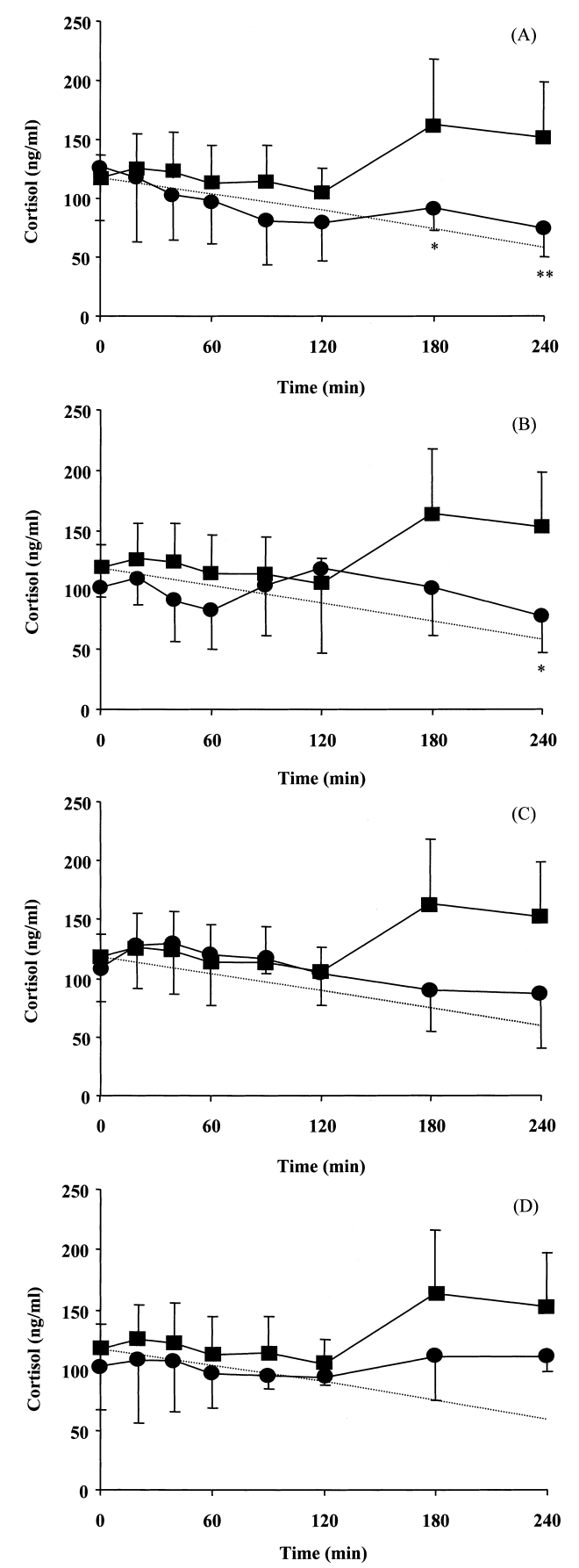

Fig. 2. Effect of Rikkunshi-to (A), Hange-shashin-to (B), Hange-kobokuto (C), and Ninjin-to (D) (O) or Placebo (ם) on Plasma Cortisol Levels

The dotted line represents the cortisol levels when blood samples were taken at intervals of $120 \mathrm{~min}$. Each point represents the mean \pm S.D. of concentrations in five volunteers. $* * p<0.01, * p<0.05$ compared with the placebo group. creases in cortisol only at 240 min (Fig. 2B), and the cortisol levels were similar to those when Rikkunshi-to was administered.

Hange-koboku-to (Fig. 2C) and Ninjin-to (Fig. 2D) had no significant effect on plasma cortisol levels. However, plasma cortisol levels at 240 min were higher with both medicines when blood was sampled at intervals of $120 \mathrm{~min}$.

\section{DISCUSSION}

The gastrointestinal function regulatory Kampo medicine Rikkunshi-to has modulatory effects on the HPA axis and autonomic nervous function. Some abnormalities of gastrointestinal function are caused by the obstruction of the HPA axis and the autonomic nervous system and by changes in hormone levels. We examined the effects of gastrointestinal function regulatory Kampo mecicines on the plasma levels of ACTH and cortisol under the stress condition.

Plasma ACTH levels are regulated by the two major pathways of circadian rhythm and negative feedback. Repetitive blood sampling raised ACTH-IS levels in plasma compared with sampling at intervals of $120 \mathrm{~min}$ in volunteers who received placebo. Those effects of placebo on ACTH are assumed to result from mental and/or physiological stress in volunteers due to repetitive blood sampling. Volunteers in whom samples were taken at intervals of $120 \mathrm{~min}$ are assumed to be under less stress. Rikkunshi-to, Hange-shashinto, and Hange-koboku-to suppressed the increases in ACTHIS levels compared with placebo, but Ninjin-to had no effect. The reasons why Ninjin-to had no effect on ACTH levels are unknown. However, the herbs contained in these Kampo medicines might play a role. In the four Kampo medicines used this study, Pinelliae Tuber is a common ingredient in the three medicines that suppressed increases in plasma ACTH levels and also has affects the central nervous system. Thus Pinelliae Tuber, or combinations of Pinelliae Tuber and Zingiberis Rhizoma (Zingiberis Siccatum Rhizoma), might play a role in controlling the increases in ACTH.

Cortisol is commonly used to indicate the level of stress. Samples taken at intervals of 120 min showed a decreasing trend in cortisol levels, corresponding with circadian rhythm. In general, plasma cortisol levels are high in the morning and gradually decrease from morning to afternoon. In our study, we found rather low plasma cortisol levels from 14:00 until 18:00, and a change in the timing of blood sampling might have yielded different results. ${ }^{19)}$ Rikkunshi-to and Hangeshashin-to suppressed increases in cortisol levels compared with placebo. Those levels showed the same circadian tendency as those of cortisol. Hange-koboku-to and Ninjin-to had no significant effect. However, Hange-koboku-to showed $54.9 \%$ (180 $\mathrm{min})$ and $56.7 \%$ (240 min) inhibition of placebo cortisol levels, and Ninjin-to showed $68.3 \%$ (180 min) and $73.5 \%$ (240 min) inhibition compared with placebo. Although further studies are needed to elucidate why only Rikkunshi-to and Hange-shashin-to had significant effects, our findings implicate pathways that influence the HPA axis.

Rikkunshi-to and Hange-shashin-to had modulatory effects on the HPA axis from the viewpoint of ACTH and cortisol. Those hormonal effects of Rikkunshi-to are confirmed in other reports. ${ }^{8,9)}$ Both medicines might influence corticotropin-releasing hormone $(\mathrm{CRH})$ or $\mathrm{ACTH}$, which exist 
upstream on the HPA axis compared with cortisol. In this study, repetitive blood sampling resulted in increases in ACTH (120, $180 \mathrm{~min})$ and cortisol (180, $240 \mathrm{~min})$ levels. Changes in plasma ACTH levels were more rapid than those in cortisol, and CRH levels might change before the changes in ACTH and cortisol. On the effects of Kampo and herbs for the HPA axis, Bupleuri Radix-containing Kampo medicines stimulate the secretion and synthesis of pituitary ACTH, and the effects are mediated by hypothalamic CRH. ${ }^{20-22)}$

Most NUD patients are exposed to continual affective stress. The continual stress causes abnormalities in the HPA axis and autonomic nervous function. Gastrointestinal function regulatory Kampo medicines have been used empirically to treat abnormalities of the gastrointestinal system such as NUD. Based on the empirical effects, some gastrointestinetargeting Kampo medicines have been elucidated from gutmotor regulatory hormone levels. The empirical effects were assumed to be due to changes in the levels of somatostatin, motilin, gastrin, or vasoactive intestinal peptide. ${ }^{23-27)}$ In addition to this mechanism, which is related to gastrointestinal regulatory hormones, Rikkunshi-to, Hange-shashin-to, and Hange-koboku-to were found to affect the HPA axis in this study. However, in our study, the effects of gastrointestinal function regulatory Kampo medicines on the HPA axis were caused by repetitive blood sampling. It is therefore necessary to examine the effects of these Kampo medicines on the HPA axis and autonomic nervous system in patients with conditions such as NUD.

In this study, Rikkunshi-to, Hange-shashin-to, and Hangekoboku-to (partially) regulated plasma ACTH and cortisol levels under stress. These modulatory effects might be beneficial in stress-related disease and the pharmacological activities of these medicines should be investigated clinically.

\section{REFERENCES}

1) Anonymous, Lancet, 1, 576-579 (1988).

2) Kok L. P., Yap I. L. E., Guan R. Y. C., Sing. Med. J., 30, 346-349 (1989).
3) Okuse S., J. Jpn. Psychosom. Soc., 15, 46-52 (1975).

4) Miyabo S., Hisada T., Asato T., Mizushima N., Ueno K., J. Clin. Endocr. Metab., 42, 1158-1162 (1976).

5) Asami T., Miyanaga K., Ida I., Miyamoto M., Higuchi T., Jpn. Pharmacol. Ther, 20, 3851-3857 (1992).

6) Oka T., Mine K., Nakagawa T., J. Med. Pharm. Soc. WAKAN-YAKU, 7, $242-245$ (1990).

7) Oka T., Nakagawa T., Matsuura T., Kogawa K., Rinsho-to-Kenkyu, 67, 243 -245 (1990).

8) Oka T., Komiyama H., Nakagawa T., Matsuura T., J. Med. Pharm. Soc. $W A K A N-Y A K U, \mathbf{8}, 360$-361 (1991).

9) Oka T., Komiyama H., Nakagawa T., Matsuura T., Oka K., Jpn. J. Orient. Med., 43, 439-446 (1993).

10) Krieger D., Recent Prog. Horm. Res., 16, 277-336 (1980).

11) Kamel A., Norgren S., Persson B., Marcus C., Arch. Dis. Child., 80, 42-45 (1999).

12) Ferriani R. A., Silva de Sa M. F., Int. J. Gynaecol. Obstet., 23, 459462 (1985).

13) Kawakami Y., Rinsho Byori, 49, 562-565 (2001).

14) Vachon P., Moreau J. P., Contemp. Top. Lab. Anim. Sci., 40, 22-24 (2001).

15) Torii R., Kitagawa N., Nigi H., Ohsawa N., Jikken Dobutsu, 42, 67 73 (1993).

16) Nagano T., Itoh H., Soeda F., Takeyama M., Jpn. J. Hosp. Pharm., 25, 257-263 (1999).

17) Kitagawa T., Shimozono T., Aikawa T., Yoshida T., Nakamura H., Chem. Pharm. Bull., 29, 1130-1133 (1981).

18) Terasaki K., Ishiwatari N., Jibiki K., Odagiri E., Demura R., Demura H., Horumon-to-Rinsho, 35, 743-749 (1987).

19) Hiroshige T., Sakakura M., Ito S., Endocr. Jpn., 16, $465-469$ (1969).

20) Iwai I., Suda T., Tozawa F., Nakano Y., Sato Y., Ohmori N., Sumitomo T., Yamada M., Demura H., Neurosci. Lett., 157, 37-40 (1993).

21) Nakano Y., Suda T., Tozawa F., Dobashi I., Sato Y., Ohmori N., Sumitomo T., Demura H., Neurosci. Lett., 160, 93-95 (1993).

22) Hiai S., Yokoyama H., Nagasawa T., Oura H., Chem. Pharm. Bull., 29, 495-499 (1981).

23) Naito T., Nagano T., Itoh H., Takeyama M., Biol. Pharm. Bull., 24, 194-196 (2001).

24) Naito T., Itoh H., Yasunaga F., Takeyama M., Biol. Pharm. Bull., 24, $841-843$ (2001).

25) Naito T., Itoh H., Yasunaga F., Takeyama M., Biol. Pharm. Bull., 25, 327-331 (2002)

26) Nagano T., Itoh H., Takeyama M., Biol. Pharm. Bull., 22, 1131-1133 (1999).

27) Nagano T., Itoh H., Takeyama M., Biol. Pharm. Bull., 23, 352-353 (2000). 